\title{
Residual Currents Induced by Asymmetric Tidal Mixing in Weakly Stratified Narrow Estuaries
}

\author{
Peng Cheng* And Arnoldo Valle-Levinson \\ Department of Civil and Coastal Engineering, University of Florida, Gainesville, Florida \\ HuIB E. DE SWART \\ Institute for Marine and Atmospheric Research Utrecht, Utrecht University, Utrecht, Netherlands
}

(Manuscript received 14 July 2009, in final form 10 May 2010)

\begin{abstract}
Residual currents induced by asymmetric tidal mixing were examined for weakly stratified, narrow estuaries using analytical and numerical models. The analytical model is an extension of the work of R. K. McCarthy, with the addition of tidal variations of the vertical eddy viscosity in the longitudinal momentum equation. The longitudinal distribution of residual flows driven by asymmetric tidal mixing is determined by the tidal current amplitude and by asymmetries in tidal mixing between flood and ebb. In a long channel, the magnitude of the residual flow induced by asymmetric tidal mixing is maximum at the estuary mouth and decreases upstream following the longitudinal distribution of tidal current amplitude. Larger asymmetry in tidal mixing between flood and ebb produces stronger residual currents. For typical tidal asymmetry, mixing is stronger during flood than during ebb and results in two-layer residual currents with seaward flow near the surface and landward flow near the bottom. For reverse tidal asymmetry, mixing is weaker during flood than during ebb and the resulting residual flow is landward near the surface and seaward near the bottom. Also, the residual flow induced by tidal asymmetry has the same order of magnitude as the density-driven flow and therefore is important to estuarine dynamics. Numerical experiments with a primitive-equation numerical model [the Regional Ocean Modeling System (ROMS)] generally support the pattern of residual currents driven by tidal asymmetry suggested by the analytical model.
\end{abstract}

\section{Introduction}

Pioneering studies of estuarine exchange flow focused on the mean effects after a tidal time scale and suggested that residual estuarine currents resulted mainly from the longitudinal baroclinic pressure gradient (Pritchard 1956; Hansen and Rattray 1965; Chatwin 1976; MacCready 2004). The influence of tidal processes was included in those theories using a constant vertical eddy viscosity that represented mixing over tidal time scales. However, it has been recognized that tides can contribute to the creation of residual currents in many estuaries through

\footnotetext{
* Current affiliation: Large Lakes Observatory, University of Minnesota, Duluth, Minnesota.

Corresponding author address: Peng Cheng, Department of Civil and Coastal Engineering, University of Florida, Gainesville, FL 32611.

E-mail: chengp@d.umn.edu
}

nonlinearities and through the temporal variation of tidal mixing. Ianniello (1977) showed that, under relatively strong turbulent mixing, the Eulerian residual currents induced by tidal nonlinearities are seaward at all depths and the Lagrangian residual currents induced by tidal nonlinearities oppose the two-layer density-driven flow. Subsequent studies have concentrated on temporal variations of tidal mixing. Jay and Smith (1990) introduced the concept of tidal asymmetry, suggesting stronger mixing during flood tides and enhanced stratification during ebb tides. They demonstrated the importance of tidal asymmetry with observations at the Columbia River.

Asymmetric tidal mixing can arise from strain-induced periodic stratification (SIPS) (Simpson et al. 1990) or extra freshwater inputs from a side embayment (Lacy et al. 2003; Fram et al. 2007). Stacey et al. (2008) used a one-dimensional numerical model to examine the tidal asymmetry caused by extra freshwater inputs and its role in the creation of estuarine subtidal circulation. They demonstrated that the residual flow generated by this 
tidal asymmetry is of the same vertical structure and magnitude as the expected estuarine gravitational circulation. They also found that the residual flow is strongly dependent on the timing of stratification beginning within the tidal cycle and developed a bulk Richardson number to predict the onset of stratification.

Tidal straining is one of the most common mechanisms for creating tidal asymmetries. The role of tidal straining in creating asymmetries in turbulent mixing at tidal time scales has been well documented through observations (Nepf and Geyer 1996; Simpson et al. 2005; Stacey and Ralston 2005; Scully and Friedrichs 2007) and numerical simulations (Simpson et al. 2002; Li et al. 2008). The strain-induced tidal asymmetry creates asymmetries in the shear of the tidal current profile, resulting in a nonzero net flow profile. During ebb tides, tidal currents stratify the water column through the straining of the density field by the interaction between a longitudinal density gradient and a vertically sheared velocity profile. During flood tides, this straining is reversed and the water column tends to be well mixed, intensifying currents near the bottom. This asymmetric mixing and velocity profile can lead to a residual flow with the same structure as the density-driven circulation: seaward flow near the surface and landward flow at the bottom (Jay and Musiak 1996; Stacey et al. 2001). However, the vertical structure of this resulting residual flow has been obtained on the basis of conceptual analysis but has not been evaluated in detail with analytical or numerical models. The purpose of this study is to investigate the spatial pattern and strength of residual currents induced by tidal-straining-induced tidal asymmetry in a two-dimensional estuary channel.

Because of the difficulty in solving the nonlinear momentum equations analytically, this study is limited to weakly stratified narrow estuaries. These can be treated as weakly nonlinear systems whose governing equations can be solved using a perturbation method. The approach is also restricted to "narrow" estuaries where lateral variability of flow and the earth's rotation can be neglected. The weakly stratified estuary is considered as an approximately well-mixed estuary. With these assumptions, the analytical model developed by McCarthy (1993) is extended to include an additional term representing tidal variations in vertical mixing. To obtain the solution, a relatively weak asymmetry in turbulent mixing between flood and ebb tides must be considered. Also, the analytical model uses an artificially designed eddy viscosity, which decouples turbulent mixing from tidal currents and stratification. Furthermore, a primitive-equation numerical model, with a two-equation turbulent closure, is also used to validate the analytical solutions.

The remainder of this paper is structured as follows: Section 2 presents a two-dimensional analytical model

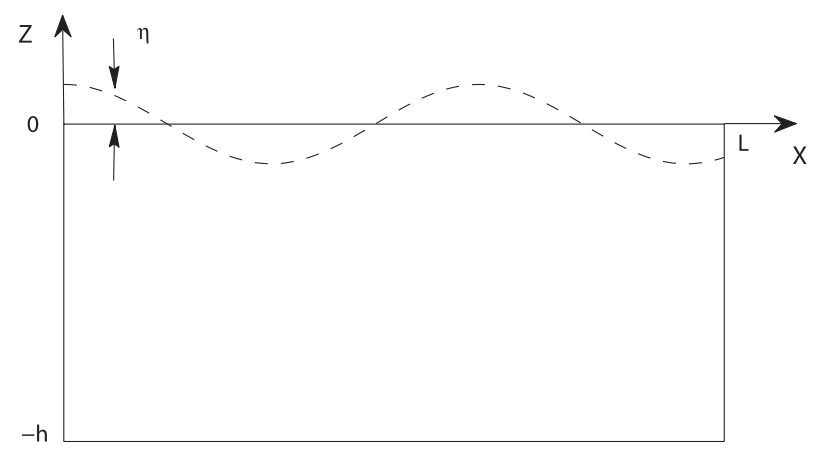

FIG. 1. Schematic of an idealized estuary channel (length not to scale): a vertical-longitudinal section is along the centerline of the channel. The dashed lines represent tidal elevations.

and the solutions of residual currents induced by tidal asymmetry. Section 3 presents a numerical experiment in an estuary channel to evaluate the generality of the analytical model and show the influence of stratification on tidal mean mixing. Finally, conclusions are presented in section 4.

\section{Analytical model}

This analytical model is an extension of the work of McCarthy (1993) by adding tidal variations of the vertical eddy viscosity in the longitudinal momentum equation. Most of the model assumptions and equation-solving techniques follow those of Ianniello (1977) and McCarthy (1993). The novel feature of this model is that asymmetric tidal mixing is included and tidal-asymmetry-induced residual currents can be explicitly computed. The model domain is a straight narrow estuary channel that has constant depth and width. Lateral variations of bathymetry are neglected so that the estuary can be simplified as a two-dimensional along-channel section. The coordinate system is shown in Fig. 1, where $x$ is positive from the mouth toward the head and $z$ is positive upward with the bottom at $z=-h$.

Here, "narrow" means that the along-channel residual dynamics are not significantly affected by lateral circulations; namely, both lateral advection and the earth's rotation can be neglected. The conditions under which these assumptions apply are given in Huijts et al. (2009) and Cheng and Valle-Levinson (2009). The first is that the Rossby number is large, which indicates that Coriolis forcing is negligible. In the case of a long estuary this implies that its width should be small compared to the Rossby radius of deformation. On the other hand, a large Rossby number suggests that lateral advection could be important in narrow estuaries, as in the Hudson River (e.g., Scully et al. 2009). Therefore, the second condition is that vertical turbulent mixing is relatively 
large, under which lateral advection is negligible. Accordingly, a narrow estuary, like the one studied here, can be considered as an estuary with large Rossby and Ekman numbers. This condition is satisfied for wellmixed and weakly stratified estuaries with small width.

\section{a. Governing equations}

The governing equations for momentum, continuity, and salt transport and the equation of state are

$$
\begin{aligned}
\frac{\partial \hat{u}}{\partial \hat{t}}+\hat{u} \frac{\partial \hat{u}}{\partial \hat{x}}+\hat{w} \frac{\partial \hat{u}}{\partial \hat{z}} & =-g \frac{\partial \hat{\eta}}{\partial \hat{x}}-\frac{g}{\hat{\rho}_{0}} \int_{\hat{z}}^{\hat{\eta}} \frac{\partial \hat{\rho}}{\partial \hat{x}} d \hat{z}+\frac{\partial}{\partial \hat{z}}\left(\hat{K} \frac{\partial \hat{u}}{\partial \hat{z}}\right), \\
\frac{\partial \hat{u}}{\partial \hat{x}}+\frac{\partial \hat{w}}{\partial \hat{z}} & =0, \\
\frac{\partial \hat{\eta}}{\partial \hat{t}}+\frac{\partial}{\partial \hat{x}}\left(\int_{-\hat{h}}^{\hat{\eta}} \hat{u} d \hat{z}\right) & =0, \\
\frac{\partial \hat{s}}{\partial \hat{t}}+\hat{u} \frac{\partial \hat{s}}{\partial \hat{x}}+\hat{w} \frac{\partial \hat{s}}{\partial \hat{z}} & =\hat{K}_{x} \frac{\partial^{2} \hat{s}}{\partial \hat{x}^{2}}+\frac{\partial}{\partial \hat{z}}\left(\hat{K} \frac{\partial \hat{s}}{\partial \hat{z}}\right),
\end{aligned}
$$

and

$$
\hat{\rho}=\hat{\rho}_{0}(1+\beta \hat{s}), \quad \beta=7.7 \times 10^{-4} .
$$

Here, a caret denotes a dimensional quantity; $\hat{t}$ is time; $\hat{u}$ and $\hat{w}$ are the longitudinal and vertical velocity components, respectively; $\hat{\eta}$ is the free surface elevation; $\hat{h}$ is the water depth; $\hat{s}$ is salinity; $\hat{\rho}$ is water density; $\hat{\rho}_{0}$ is freshwater density; $\hat{\beta}$ is the haline contraction coefficient; $\hat{K}_{x}$ is the horizontal eddy diffusivity; and $\hat{K}$ represents vertical eddy viscosity and diffusivity, assuming the Schmidt number (ratio of eddy viscosity to diffusivity) is 1 . The above equations are the same as those used by McCarthy (1993) except that the salt balance is used here instead of density.

To include asymmetric tidal mixing in the dimensionless equations, we assume that the vertical turbulent mixing coefficient can be decomposed in two parts:

$$
\hat{K}=\hat{K}^{*}+\hat{K}^{\prime} .
$$

Here $\hat{K}^{*}$ measures the degree of mixing that would occur under well-mixed conditions, whereas $\hat{K}^{\prime}$ represents a tidal variation part caused by tidal straining. Ianniello (1977) argues that in a tidally dominated, well-mixed estuary

$$
\hat{K}^{*}=C_{d}|\hat{U}| \hat{h} \hat{f}(\hat{z}),
$$

where $C_{d}$ is a drag coefficient, $\hat{U}$ the leading order depth-averaged tidal velocity, and $\hat{f}(\hat{z})$ a function of the vertical coordinate $\hat{z}$; we assume $\hat{U}$ is a constant.
A similar perturbation technique to Ianniello's (1977) and McCarthy's (1993) is followed. The subsequent scalings are introduced:

$$
\begin{aligned}
& t=\hat{t} \omega, \quad x=\frac{\hat{x}}{\sqrt{g \hat{h} / \omega}}, \quad z=\frac{\hat{z}}{\sqrt{2 K_{0} / \omega}}, \\
& d=\frac{\hat{h}}{\sqrt{2 K_{0} / \omega}}, \quad \eta=\frac{\hat{\eta}}{\eta^{*}}, \\
& u=\frac{\hat{u}}{\eta^{*} \sqrt{g / \hat{h}}}, \quad w=\frac{\hat{w}}{\eta^{*} \sqrt{2 K_{0} \omega / \hat{h}}}, \quad s=\frac{\hat{s}}{s_{c}}, \quad K=\frac{\hat{K}}{K_{0}},
\end{aligned}
$$

and

$$
K_{x}=\frac{\hat{K}_{x}}{g \eta^{* 2} / \hat{h} \omega}
$$

in which the variables without a caret are dimensionless quantities. Here $K_{0}=C_{d} \hat{U} \hat{h} \hat{f}(\hat{z})$ is a constant vertical eddy viscosity, $\eta^{*}$ is the tidal amplitude at the mouth, $s_{c}$ is the ocean salinity, $\omega$ is the angular frequency of tidal forcing, and $d$ is a measurement of the depth in terms of the frictional length scale $\sqrt{2 K_{0} / \omega}$ (note that $1 / d$ is analogous to the Ekman number); the horizontal length scale is the tidal wavelength $\sqrt{g \hat{h}} / \omega$. Substituting these scalings into Eq. (1) results in the dimensionless equations:

$$
\begin{aligned}
\frac{\partial u}{\partial t}+\varepsilon u \frac{\partial u}{\partial x}+\varepsilon w \frac{\partial u}{\partial z} & =-\frac{\partial \eta}{\partial x}-\varepsilon \gamma \int_{z}^{\varepsilon \eta d} \frac{\partial s}{\partial x} d z+\frac{1}{2} K \frac{\partial^{2} u}{\partial z^{2}} \\
\frac{\partial u}{\partial x}+\frac{\partial w}{\partial z} & =0
\end{aligned}
$$

$\frac{\partial \eta}{\partial t}+\frac{1}{d} \frac{\partial}{\partial x}\left(\int_{-d}^{\varepsilon \eta d} u d z\right)=0$

and

$$
\frac{\partial s}{\partial t}+\varepsilon u \frac{\partial s}{\partial x}+\varepsilon w \frac{\partial s}{\partial z}=\varepsilon^{2} K_{x} \frac{\partial^{2} s}{\partial x^{2}}+\frac{1}{2} \frac{\partial}{\partial z}\left(K \frac{\partial s}{\partial z}\right),
$$

where $\varepsilon=\eta^{*} / \hat{h}$ and $\gamma=\beta s_{c} / \epsilon d$. Of particular interest is the nonlinear effect represented by the tidal amplitude to depth ratio $\varepsilon$. The assumption is that the parameter $\varepsilon$ is not negligible but is much smaller than 1 . Since $\varepsilon \ll 1$, the nonlinear advection terms do not affect the tidal solution and $\varepsilon$ becomes the expansion parameter for the dependent variables. The parameter $\gamma$ measures the relative importance of the baroclinic pressure gradient and 
is assumed $O(1)$. As McCarthy (1993) discussed, if $\gamma \sim O(1)$, the estuary is dominated by barotropic tides, stratification is weak, and the baroclinic forcing term mainly contributes to residual currents. The dimensionless set of equations is the same as those of McCarthy.

The dimensionless vertical mixing coefficient is written as

$$
K=K^{*}+\varepsilon K^{\prime} ; \quad K^{*}=\frac{\hat{K}^{*}}{K_{0}} ; \quad K^{\prime}=\frac{\hat{K}^{\prime}}{\varepsilon K_{0}} .
$$

Herein,

$$
K^{*}=f(z)
$$

with $f(z)=\hat{f}(\hat{z})$, and $K^{\prime}(z, t)$ depends on the vertical coordinate $z$ and time $t$ in a user-specified manner. Variations in turbulent vertical mixing owing to tidal straining are assumed to be small compared to turbulent mixing induced by frictional drag at the bottom. Moreover, it is assumed that $f(z)=1$; that is, the turbulent mixing coefficient $K^{*}$ does not depend on the vertical coordinate. In many previous analytical studies, $K$ is assumed constant over a tidal cycle. In this study, we use two constants that represent eddy viscosities during flood and ebb tides (Fig. 2). The difference between the two constants translates into asymmetric tidal mixing. This assumption uses the flood-averaged mixing to represent the entire flood and uses the ebb-averaged mixing to represent the entire ebb. This could be the simplest case of asymmetric tidal mixing. More complicated tidal variations of mixing must await future studies. It is noteworthy that $K^{*}$ can be chosen as the tidally averaged eddy viscosity or a value smaller than the eddy viscosity of ebb tides, as shown in Fig. 2. Although the two choices produce similar results, the latter is preferred because the mean of the tidal variation component is not zero, consistent with the idea that tidal asymmetry produces "extra" eddy viscosity over a tidal cycle. The ratio between the variable and the steady components of $K$ is assumed to be $O(\varepsilon)$. This indicates a weakly stratified estuary where variations of vertical mixing induced by stratification are comparatively small. With this assumption, asymmetric tidal mixing only influences residual currents.

Following the perturbation method, the dependent variables $\eta, u, w$, and $s$ are expanded in an asymptotic series in powers of $\varepsilon$, yielding the lowest- $\left[O\left(\varepsilon^{0}\right)\right]$ and the first- $\left[O\left(\varepsilon^{1}\right)\right]$ order perturbation equations. The lowest order equations represent tidal motion and are the same as those of Ianniello (1977), and the governing equations of salt transport are the same as those of McCarthy (see appendix). Solutions to tidal motion and salinity have

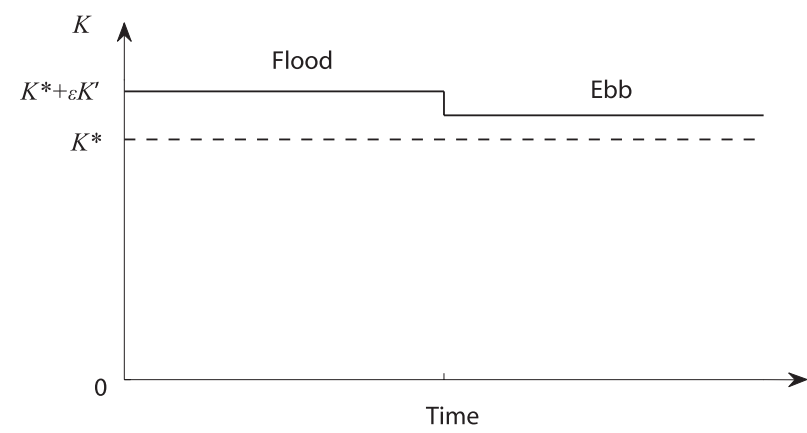

FIG. 2. Tidal evolution of dimensionless eddy viscosity $K ; K^{*}$ is the steady component, while $\varepsilon K^{\prime}$ represents a tidal variation component of $K$.

been provided by Ianniello (1977) and McCarthy (1993), respectively, and are omitted here. This study concentrates on the residual currents only. Time averaging the first-order momentum and continuity equations after a tidal cycle gives

$$
\begin{aligned}
\overline{u_{0} \frac{\partial u_{0}}{\partial x}}+\overline{w_{0} \frac{\partial u_{0}}{\partial z}}= & -\frac{\partial \bar{\eta}_{1}}{\partial x}+\gamma \frac{d s_{0}}{d x} z+\frac{1}{2} \frac{\partial^{2} \bar{u}_{1}}{\partial z^{2}} \\
& +\frac{1}{2} \frac{\partial}{\partial z} \overline{\left(K^{\prime} \frac{\partial u_{0}}{\partial z}\right)}
\end{aligned}
$$

and

$$
\frac{1}{d} \int_{-d}^{0} \bar{u}_{1} d z+\overline{\left.u_{0}\right|_{z=0} \eta_{0}}=-R
$$

where subscripts 0 and 1 represent lowest and first order, respectively. The overbar denotes tidal averages, and $R$ is the dimensionless river flow velocity, which is the ratio of the dimensional river flow velocity $\hat{u}_{r}$ to the scale of the nonlinear flow (McCarthy 1993); namely, $R=$ $\hat{u}_{r} /\left(\varepsilon^{2} \sqrt{g \hat{h}}\right)$. The residual currents $\bar{u}_{1}$ are considered to have four components: (i) the tidally rectified flow $\bar{u}_{1 N}$, (ii) the river-induced flow $\bar{u}_{1 R}$, (iii) the density-driven flow $\bar{u}_{1 D}$, and (iv) the flow induced by asymmetric tidal mixing $\bar{u}_{1 A}$. The present model setup implies that the four components are linearly independent. Thus, $\bar{u}_{1}$ and the residual elevation $\bar{\eta}_{1}$ can be written as

$$
\bar{u}_{1}=\bar{u}_{1 N}+\bar{u}_{1 R}+\bar{u}_{1 D}+\bar{u}_{1 A}
$$

and

$$
\bar{\eta}_{1}=\bar{\eta}_{1 N}+\bar{\eta}_{1 R}+\bar{\eta}_{1 D}+\bar{\eta}_{1 A}
$$

Substituting Eqs. (11) into (10), we obtain five equations corresponding to the four sources of residual currents: 


$$
\begin{aligned}
& \overline{u_{0} \frac{\partial u_{0}}{\partial x}}+\overline{w_{0} \frac{\partial u_{0}}{\partial z}}=-\frac{\partial \bar{\eta}_{1 N}}{\partial x}+\frac{1}{2} \frac{\partial^{2} \bar{u}_{1 N}}{\partial z^{2}}, \\
& 0=-\frac{\partial \bar{\eta}_{1 R}}{\partial x}+\frac{1}{2} \frac{\partial^{2} \bar{u}_{1 R}}{\partial z^{2}}, \\
& 0=-\frac{\partial \bar{\eta}_{1 D}}{\partial x}+\gamma \frac{d s_{0}}{d x} z+\frac{1}{2} \frac{\partial^{2} \bar{u}_{1 D}}{\partial z^{2}}, \\
& 0=-\frac{\partial \bar{\eta}_{1 A}}{\partial x}+\frac{1}{2} \frac{\partial^{2} \bar{u}_{1 A}}{\partial z^{2}}+\frac{1}{2} \frac{\partial}{\partial z}\left(\overline{K^{\prime} \frac{\partial u_{0}}{\partial z}}\right),
\end{aligned}
$$

and

$$
\begin{aligned}
& \frac{1}{d} \int_{-d}^{0} \bar{u}_{1 N} d z+\frac{1}{d} \int_{-d}^{0} \bar{u}_{1 R} d z+\frac{1}{d} \int_{-d}^{0} \bar{u}_{1 D} d z \\
& +\frac{1}{d} \int_{-d}^{0} \bar{u}_{1 A} d z+\overline{\left.u_{0}\right|_{z=0} \eta_{0}}=-R
\end{aligned}
$$

\section{b. Solution of residual currents induced by asymmetric tidal mixing}

The four components of residual currents can be obtained by solving Eqs. (10). The tidally rectified flow [Eq. (10a)] has been solved by Ianniello $(1977,1981)$. The solutions of river-induced flow [Eq. (10b)] and density-driven flow [Eq. (10c)] have been provided by McCarthy (1993). The aim of this study is to obtain the solution of the flow induced by asymmetric tidal mixing. The boundary conditions used to solve the momentum equation [Eq. (10d)] include no slip at the bottom and no shear at the surface. The continuity equation for flow induced by asymmetric tidal mixing can be obtained from Eq. (10e). According to Ianniello (1977), $d^{-1} \int_{-{ }^{d}}^{0} \bar{u}_{1 N} d z+\overline{\left.u_{0}\right|_{z=0} \eta_{0}}=0$. Also, it is known that $d^{-1} \int_{-d}^{0} \bar{u}_{1 R} d z=-R$ and $d^{-1} \int_{-d}^{0} \bar{u}_{1 D} d z=0$ (McCarthy 1993). Thus, the continuity equation [Eq. (10e)] can be reduced to $d^{-1} \int_{-d}^{0} \bar{u}_{1 A}=0$. On the basis of those conditions, the solution of the flow induced by tidal asymmetry [integrating Eq. (10d) twice and using continuity] is

$$
\bar{u}_{1 A}=\frac{\partial \bar{\eta}_{1 A}}{\partial x}\left(z^{2}-d^{2}\right)-\int_{-d}^{z} \overline{K^{\prime} \frac{\partial u_{0}}{\partial z^{\prime}}} d z^{\prime}
$$

and

$$
\frac{\partial \bar{\eta}_{1 A}}{\partial x}=\frac{-3}{2 d^{3}} \int_{-d}^{0} \int_{-d}^{z} \overline{K^{\prime} \frac{\partial u_{0}}{\partial z^{\prime}}} d z^{\prime} d z
$$

In which $z^{\prime}$ is a dummy variable. The solution shows that the residual flow induced by tidally asymmetric mixing is determined by the lowest order tidal current $u_{0}$ and the

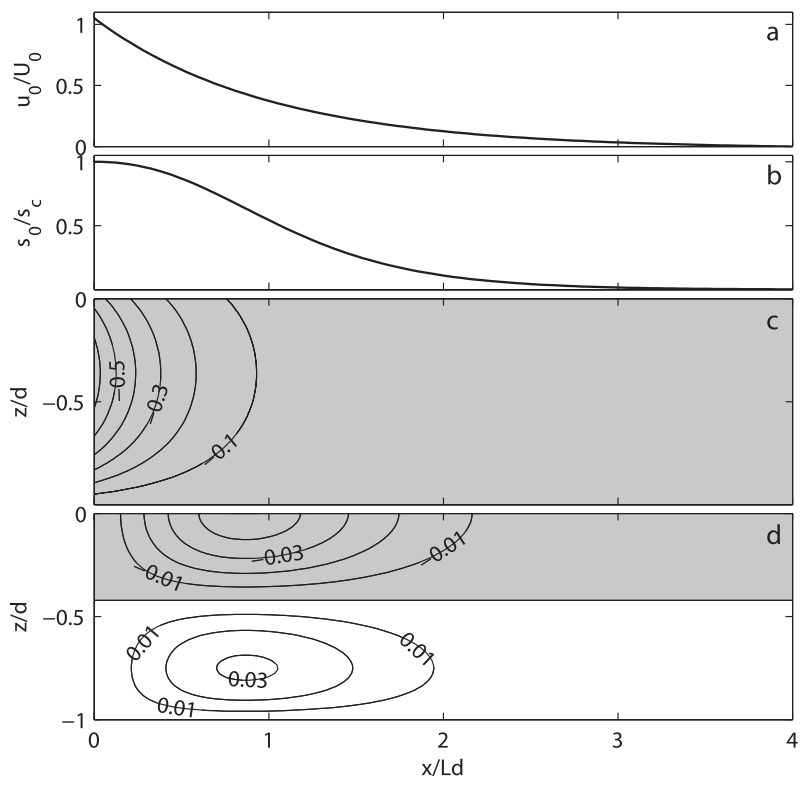

FIG. 3. Dimensionless analytical solutions for a long channel: (a) amplitude of tidal current $u_{0}$ scaled by $U_{0}$ that equates $\varepsilon \sqrt{g h}$; (b) scaled lowest order salinity $s_{0}$, where $s_{c}$ is the ocean salinity; (c) residual tidally rectified currents; and (d) density-driven currents. Darker area denotes negative values (outflow), and $L_{d}$ is a tidal dissipation length. Scaled parameters are $\varepsilon=0.1, d=1$, $R=0.1, L_{d}=1.65$, and $K_{x}=0.09$. The solutions follow Ianniello $(1977,1981)$ and McCarthy (1993).

tidal variation of vertical mixing $K^{\prime}$. The solution for $u_{0}$ has been given by Ianniello (1977). A proper form of vertical eddy viscosity needs to be designed so as to examine the pattern and strength of $\bar{u}_{1 A}$. As assumed, $K^{*}$ is a steady part and has no vertical distribution; $K^{\prime}$ has both temporal and vertical variations. It is constant throughout the flood or ebb but has a different magnitude for each phase of the tidal cycle (Fig. 2). With stratification, the vertical profile of eddy viscosity tends to show an asymmetric parabolic distribution with the maximum value moving toward the bottom (Businger et al. 1971). Therefore, the vertical profile of $K^{\prime}$ is designed as an exponential function $K^{\prime}(z)=(d+z) /$ $\varepsilon \exp \left[-(d+z) / a_{0}\right]$, which is controlled by the parameter $a_{0}$. Once $K^{\prime}$ is determined, $\bar{u}_{1 A}$ is obtained by integrating Eq. (11) numerically.

Taking advantage of the tidal solution from Ianniello (1977), the residual currents induced by tidally asymmetric mixing are obtained for an infinitely long channel. The solution for a channel with a reflecting wall has a similar pattern to the infinitely long channel and will not be presented here. Figure 3 shows longitudinal distributions of tidal current amplitude $u_{0}$, depth- and time-mean salinity $s_{0}$, tidal-nonlinearity-induced residual currents $\bar{u}_{1 N}$, and density-driven currents $\bar{u}_{1 D}$ for a long 

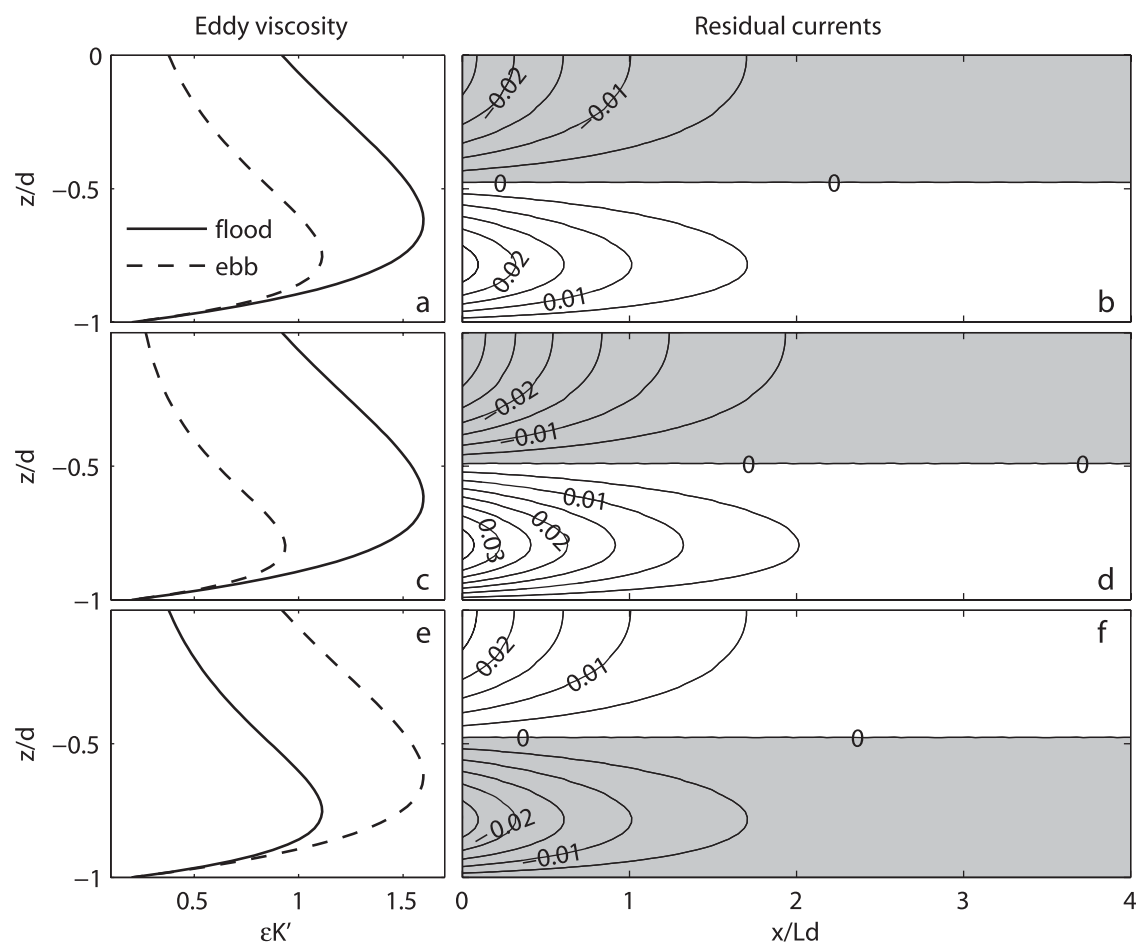

FIG. 4. Dimensional solutions of residual currents induced by asymmetric tidal mixing: (left) vertical profiles of eddy viscosity for flood and ebb tides and (right) an axial section of residual currents induced by asymmetric tidal mixing; (top) case 1, a typical tidal asymmetry; (middle) case 2 , a typical tidal asymmetry with larger asymmetry of eddy viscosity between flood and ebb tides, relative to case 1; and (bottom) reverse tidal asymmetry. The scaled parameters are as in Fig. 3. Negative values (shaded) denote seaward flow.

channel. The values of the dimensional variables were chosen as $\eta^{*}=1 \mathrm{~m}, \hat{h}=10 \mathrm{~m}, \hat{u}_{r}=0.01 \mathrm{~m} \mathrm{~s}^{-1}$, and $\hat{\omega}=$ $7.03 \times 10^{-5} \mathrm{~s}^{-1}\left(M_{2}\right.$ tide $)$. The scaled parameters are $\varepsilon=$ $0.1, d=1, R=0.1, L_{d}=1.65$, and $K_{x}=0.09$, where $L_{d}$ is a tidal dissipation length scale defined by Ianniello (1977). The parameter $d$ indicates mixing effects and a value of 1.0 represents a vertical eddy viscosity $K_{0}=$ $0.007 \mathrm{~m}^{2} \mathrm{~s}^{-1}$ (relatively strong mixing). The scaled tidal current amplitude decreases from about 1.0 at the mouth to 0 at the head of the estuary because of friction (Fig. 3a). The salinity is scaled by seawater salinity such that a value of one indicates seawater and a scaled salinity of zero represents freshwater. The salinity is one at the mouth and approaches zero upstream (Fig. 3b). The maximum horizontal salinity gradient occurs near $x=L_{d}$. Tidalnonlinearity-induced residual currents are seaward at all depths and decrease from the mouth toward the head and from surface to bottom (Fig. 3c). The maximum tidalnonlinearity-induced currents are concentrated in the upper water column and at the mouth. The density-driven flow shows a two-layer structure, that is, outflow near the surface and inflow near the bottom (Fig. 3d). The maximum flow is located at the region of maximum horizontal salinity gradient and is consistent with conventional estuarine dynamics theory (e.g., Hansen and Rattray 1965; Chatwin 1976; Officer 1976). The strength of the density-driven flow is smaller than the tidally induced residual currents so that density-driven flow is relatively less important in well-mixed or weakly stratified tidal estuaries.

On the basis of the solution for $u_{0}$, three cases of $K^{\prime}$ and $\bar{u}_{1 A}$ are examined (Fig. 4). The first case tests "typical" asymmetric tidal mixing, that is, stronger vertical mixing during flood than on ebb tides (Figs. $4 a, b)$. The depth mean of $K^{\prime}$ is 1.25 during flood and 0.75 during ebb. The tidal average of the depth mean of $K^{\prime}$ is 1 so that it fulfills the requirement that $K^{\prime}$ has a magnitude of $O(1)$, just as $K^{*}$. The residual currents induced by asymmetric tidal mixing show a two-layer structure with seaward flow near the surface and landward flow near the bottom. This is similar to the density-driven flow, but the maximum magnitude of $\bar{u}_{1 A}$ appears at the mouth of the estuary. Clearly $\bar{u}_{1 A}$ is partly determined by tidal currents, and the strength of $\bar{u}_{1 A}$ follows the longitudinal distribution of tidal current amplitude, which decreases landward. 
The second case examines larger asymmetric mixing between flood and ebb tides: $K^{\prime}$ is the same as that in the first case during flood but is weaker during ebb (Fig. 4c). The distribution of $\bar{u}_{1 A}$ is still a two-layer structure similar to the first case, but the magnitude is stronger (Fig. 4d). This shows that a larger asymmetry of mixing between flood and ebb produces stronger residual currents. The third case tests "reverse" asymmetric tidal mixing; namely, mixing during flood is weaker than during ebb. The magnitude of $K^{\prime}$ in the third case is opposite to that in the first case; that is, $K^{\prime}$ at flood is the same as that during ebb in the first case (Fig. 4e). As in the first case, $\bar{u}_{1 A}$ has similar magnitude but exhibits the opposite pattern of landward flow near the surface and seaward currents near the bottom layer (Fig. 4f). This indicates that reverse asymmetric tidal mixing produces an opposite pattern of residual currents to the typical tidal asymmetry. It is noteworthy that the magnitude of $\bar{u}_{1 A}$ is of the same order as that of the density-driven flow, so they will compete to determine the residual flows.

Three types of reverse tidal asymmetry can be identified on the basis of previous studies. First, reverse tidal asymmetry can be created by a freshwater source from a side embayment of the estuary (Lacy et al. 2003; Fram et al. 2007). When less dense waters from a side embayment move into the estuary channel during flood tides, stratification can be stronger during the flood than the ebb, resulting in reverse tidal asymmetry. Second, most estuaries have lateral bathymetric variations. The deep channel and shoals may undergo opposite tidal evolution of stratification. At the thalweg, the water column exhibits a typical tidal asymmetry due to tidal straining; while over the shoals the water column shows a reverse tidal asymmetry. Lateral straining enhances stratification during flood tides, whereas vertical mixing reduces stratification during ebb tides (Cheng et al. 2009). Third, reverse tidal asymmetry can appear in the region of the axis of varying stratification (Fugate et al. 2007). This usually happens at the upper reaches of the estuarine salt intrusion. During flood tides, stratified water is advected upstream and the water column becomes stratified. During ebb tides, the water column is well mixed as freshwater that extends from surface to bottom moves downstream.

The phasing of stratification has been demonstrated to be important in the creation of tidal-asymmetry-induced residual currents (Stacey et al. 2008). To examine the effects of phasing of stratification on residual currents, we carried out a series of experiments following case 1 . We assume that half of the tidal cycle is well mixed, represented by a relatively large eddy viscosity (solid line in Fig. 4a), and that the other half of the tidal cycle is weakly stratified, represented by a small eddy

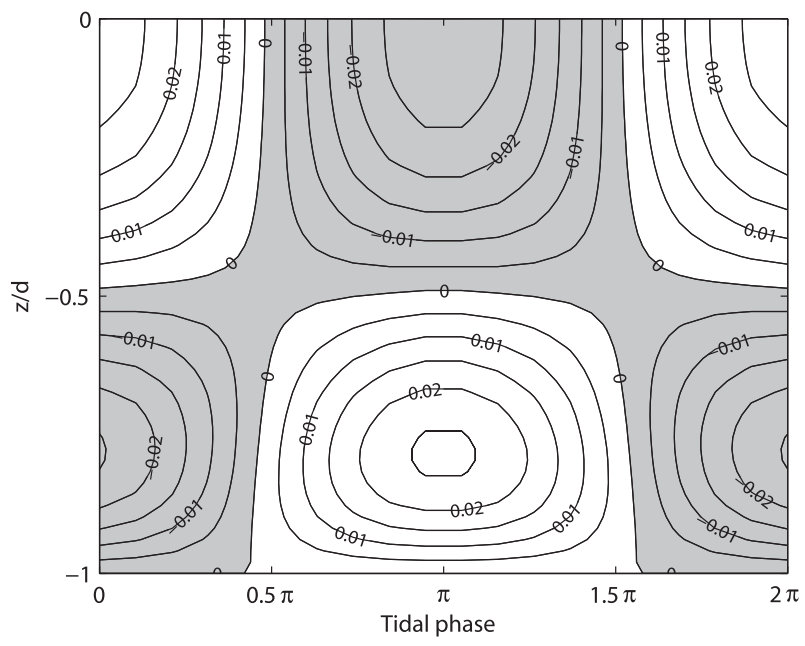

FIG. 5. Tidal-asymmetry-induced residual currents as a function of tidal phase at which stratification starts. The horizontal axis is the timing of the onset of stratification (phase of the tidal cycle): $0(\pi)$ represents the beginning of the flood (ebb). It is assumed that half of the tidal cycle is stratified. Vertical axis is dimensionless depth. Profiles are near the estuary mouth. Contours show dimensionless magnitude of the residual currents. Negative values (shaded) denote seaward flow.

viscosity (dashed line in Fig. 4a). The onset of stratification can occur at any stage of the tidal cycle. Stratification starting at the beginning of flood represents stratified flood and well-mixed ebb, that is, reverse tidal asymmetry. When stratification starts at the beginning of ebb, it represents stratified ebb and well-mixed flood, that is, typical tidal asymmetry. A station near the estuary mouth is selected to show the vertical structure of tidal-asymmetry-induced residual currents for each tidal phase of stratification onset (Fig. 5). When the entire flood is well mixed (at phase of $\pi$ ) or stratified (at phase of 0 or $2 \pi$ ), the residual currents are strongest and have opposite sign. When half of the flood is stratified (at phases of $\pi / 2$ and $3 \pi / 2$ ), the tidal-asymmetry-induced residual currents are essentially zero. When the onset of stratification occurs from $\pi / 2$ to $3 \pi / 2$, the residual currents show a similar pattern to that of density-driven circulation. When the onset of stratification occurs in the rest of the tidal cycle (at phases of $0-\pi / 2$ or $3 \pi / 2-2 \pi$ ), the residual currents show an opposite pattern to the density-driven flow. These results are consistent with those of Stacey et al. (2008).

\section{Numerical experiment}

The analytical model demonstrated that residual flow can be produced from asymmetric tidal mixing and revealed its vertical structure. However, the generality of the analytical results may be jeopardized by its 


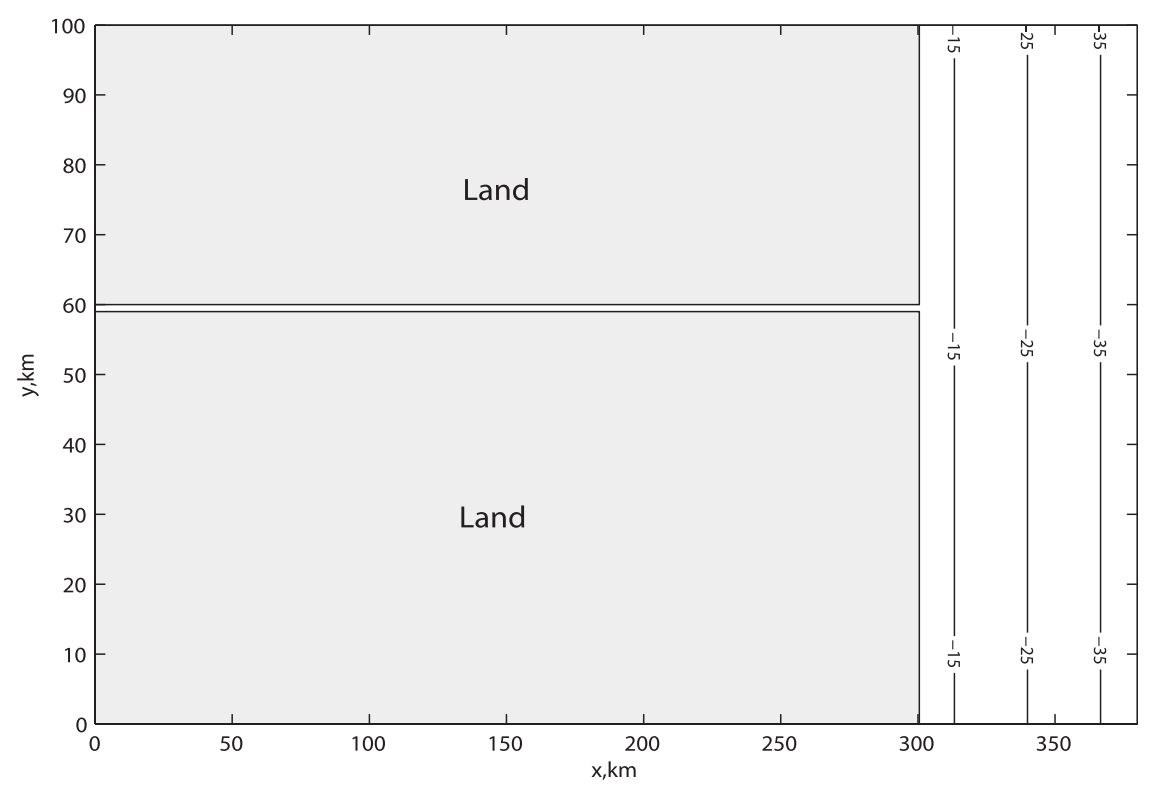

FIG. 6. Model domain of the numerical model. Contours denote water depth on the shelf.

assumptions. The analytical model is built for wellmixed estuaries (McCarthy 1993) for which the tidal variation of vertical mixing has to be assumed small. The eddy viscosity is artificially prescribed and is not coupled to tidal currents and stratification. To overcome these limitations of the analytical model, a primitive-equation numerical model with a more realistic turbulence model is required. The main purpose of the numerical experiment is to examine the validity of the analytical results instead of carrying out a comprehensive numerical study for all types of estuaries. The numerical experiment focuses on weakly stratified, narrow estuaries: partially mixed and highly stratified estuaries will be addressed in a future study.

\section{a. Model configuration}

The Regional Ocean Modeling System (ROMS) is used to carry out idealized experiments. The model is a free-surface, hydrostatic, primitive-equations ocean model that uses stretched, terrain-following vertical coordinates and orthogonal curvilinear horizontal coordinates on an Arakawa C grid (Haidvogel et al. 2000). The model domain is designed as an estuary-shelf system (Fig. 6) following the study of Hetland and Geyer (2004). The part of the domain corresponding to the estuary is straight, $300 \mathrm{~km}$ long, and has no along-channel bottom slope. The cross-channel section has a rectangular shape with a depth of $10 \mathrm{~m}$. The width is $0.6 \mathrm{~km}$. A freshwater discharge with fixed section-averaged velocity of $0.01 \mathrm{~m} \mathrm{~s}^{-1}$ is specified at the head of the estuary. The inflowing river water is prescribed to have zero salinity and temperature $15^{\circ} \mathrm{C}$, identical to the background temperature set throughout the entire domain. The continental shelf is $80 \mathrm{~km}$ wide and has a fixed cross-shelf slope of $0.05 \%$. A semidiurnal tide $S_{2}$ with an amplitude of $1.5 \mathrm{~m}$ is imposed at the eastern open boundary. The salinity of the coastal ocean is $35 \mathrm{psu}$, and a southward weak flow $\left(0.03 \mathrm{~m} \mathrm{~s}^{-1}\right)$ is specified on the shelf to suppress the bulge of freshwater at the estuary mouth. The coastal ocean is included in the domain to avoid specifying boundary conditions at the estuary mouth, which are usually difficult to establish. The two-equation turbulence closure $k-\omega$ is used to calculate vertical mixing.

The model grid is 200 (along channel, $x$ direction) by 80 (cross channel, $y$ direction) by 40 (vertical, $z$ direction) cells. The river has 150 grid cells along the channel and 3 grid cells across the channel. The along-channel grid size $(\Delta x)$ increases exponentially from the estuary mouth $(\sim 50 \mathrm{~m})$ to its head $(\sim 11 \mathrm{~km})$, providing a highly resolved region near the mouth. The cross-channel grid in the estuary is uniformly distributed and the vertical layers are uniformly discretized. The model runs from rest, for 70 days until reaching steady state. The results of the last day are used for analysis.

\section{b. Residual currents induced by asymmetric tidal mixing}

Figure 7 shows the along-channel distribution of the tidally averaged salinity field. The vertical distributions of salinity indicates that the estuary is approximately well mixed at the upper section $(x<270 \mathrm{~km})$ and is weakly stratified at the lower section $(x>270 \mathrm{~km}$ to the 


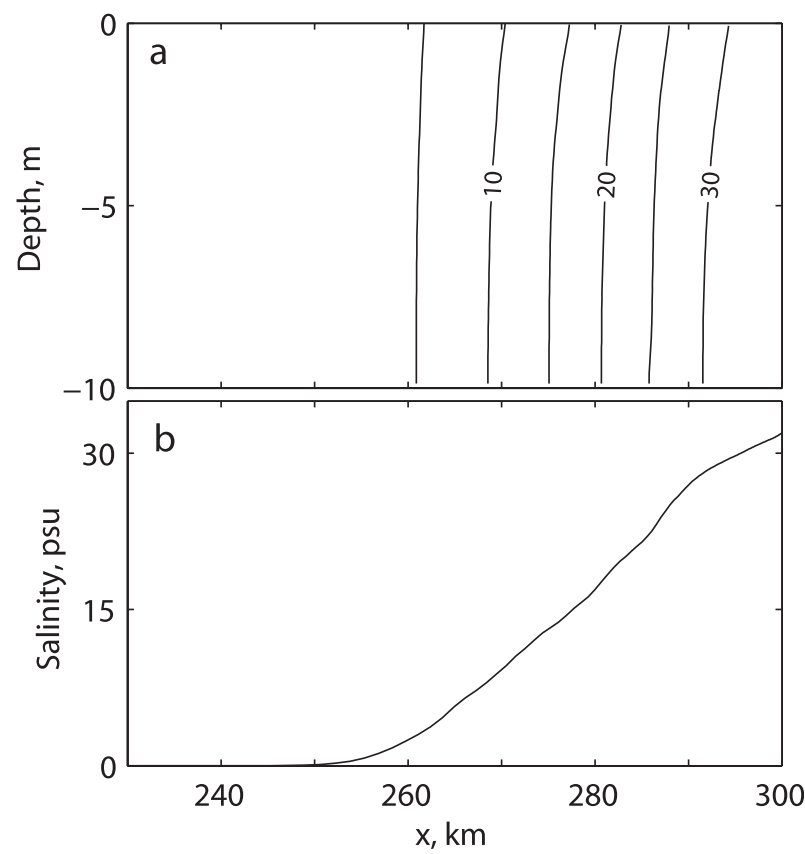

FIG. 7. Longitudinal distribution of tidally averaged salinity from the numerical model: (a) axial section of salinity in the estuarine region and (b) depth-mean salinity.

mouth). The tidal evolution of the salinity field (not shown) exhibits a well-mixed flood tide and a weakly stratified ebb tide. The longitudinal distribution of tidally averaged depth-mean salinity (Fig. 7b) shows the general form of the hyperbolic tangent function observed in many coastal plain estuaries (Pritchard 1952; Hansen and Rattray 1965). Salinity increases downstream and gradually reaches the terminal value of the ocean. The maximum horizontal salinity gradient occurs around $x=290 \mathrm{~km}$. Near the estuary mouth $(290$ $300 \mathrm{~km}$ ), the horizontal salinity gradient decreases and the curvature of the salinity curve becomes negative. This may be attributed to the strong tidal dispersion near the estuary mouth (McCarthy 1993; MacCready 2004). According to the salinity distribution, we consider that the "inner regime" of the estuary is located at 240 $270 \mathrm{~km}$ and is approximately well mixed. The "central regime" is located at $270-290 \mathrm{~km}$, and the "outer regime" is located at $290-300 \mathrm{~km}$. Both central and outer regimes are weakly stratified.

Longitudinal distributions of depth-mean along-channel tidal current amplitude and vertical eddy viscosity, averaged during flood and ebb tides (Fig. 8), show distinct distributions. For both tidal phases the tidal current amplitude, obtained by harmonic analysis, decreases from the mouth and becomes nearly zero at the head. The flood-averaged velocity is larger than the ebb-averaged velocity near the head $(0-100 \mathrm{~km})$ and is smaller than

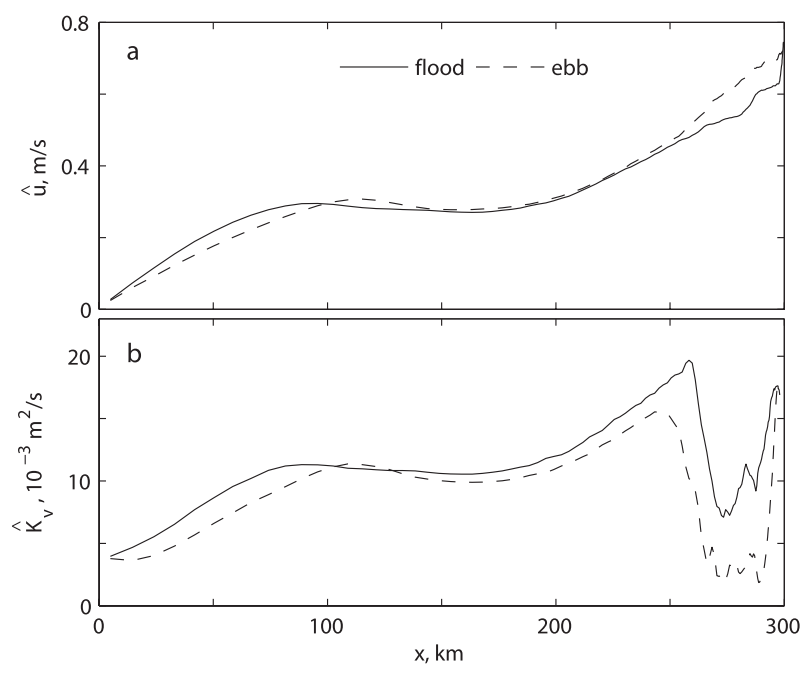

FIG. 8. Longitudinal distributions of (a) depth-mean tidal current amplitude and (b) depth-mean eddy viscosity from the numerical model. Solid (dashed) lines represent flood (ebb) averages.

the ebb-averaged velocity near the mouth (150-300 km). Between 0 and $240 \mathrm{~km}$, where river flow dominates (zero salinity), eddy viscosities are proportional to tidal current amplitudes and show similar longitudinal distributions to the velocities. In the estuary $(x>240 \mathrm{~km})$, however, the ebb-averaged eddy viscosity is much smaller than the flood-averaged eddy viscosity, showing an opposite trend as the tidal current amplitudes. This indicates a typical asymmetric tidal mixing.

The tidal-asymmetry-induced flow is calculated using a dimensional form of Eq. (11) in which variables are taken from the numerical model output. Here $\hat{K}^{*}$ is chosen as the tidally averaged depth-mean eddy viscosity, $\hat{K}^{\prime}$ is the tidal fluctuation component of the eddy viscosity (i.e., $\hat{K}^{\prime}=\hat{K}-\hat{K}^{*}$ ), and $u_{0}$ is the modeled tidal current amplitude. The tidal-asymmetry-induced flow exhibits a two-layer structure with seaward flow near the surface and landward flow near the bottom (Fig. 9). The pattern is consistent with the predicted residual flow induced by typical tidal asymmetry from the analytical model (e.g., Fig. 4b). The maximum residual flow appears in the central regime of the estuary instead of at the estuary mouth as deduced by the analytical model. This discrepancy may partly result from the alongestuary distribution of $\hat{K}^{*}$, which is assumed constant through the estuary in the analytical model. In contrast, modeled eddy viscosities show that $\hat{K}^{*}$ is reduced in the central regime of the estuary (Fig. 10a) and, according to Eq. (11a), small $\hat{K}^{*}$ results in stronger residual currents. The numerical results also suggest that the analytical model has potential utility in real estuaries if proper information of the eddy viscosity and tidal currents is available. 


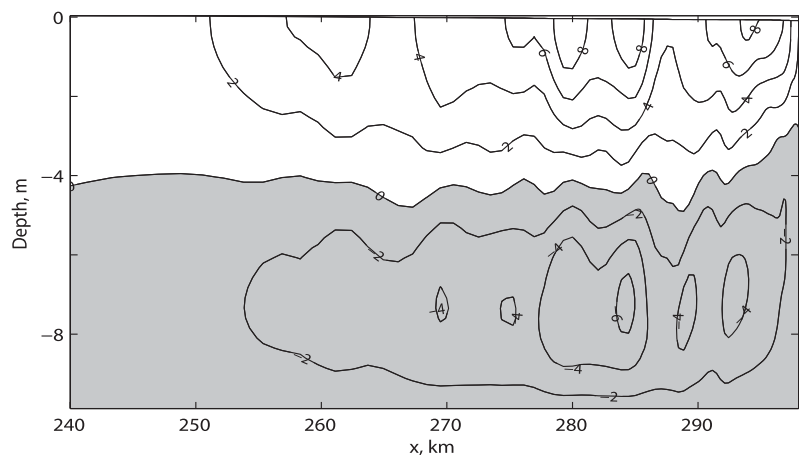

FIG. 9. Longitudinal distribution of tidal-asymmetry-induced flow of the numerical model. The unit of the residual flow is $\mathrm{cm} \mathrm{s}^{-1}$. Negative values (shaded) denote landward flow.

\section{c. Tidally averaged eddy viscosity in the central regime of the estuary}

The numerical model shows a peculiar feature of turbulent mixing. The eddy viscosities are markedly reduced and are approximately constant throughout the central regime of the estuary (Fig. 10a). This is consistent with the assumption of the analytical theory of Hansen and Rattray (1965), but the cause of this spatial distribution (constant) is unclear. In general, turbulent mixing can be parameterized in terms of a velocity scale and a length scale. The velocity scale is usually the tidal current velocity and the length scale can be taken as the water depth of the channel (MacCready 2007). This scale, however, does not account for the influence of stratification on turbulent mixing and is only proportional to the tidal current amplitude because of the uniform depth of the estuary. The amplitude of tidal currents, obtained by harmonic analysis, decreases landward almost linearly (Fig. 10b). Obviously, this scale is not able to predict the constant eddy viscosity in the central regime of estuary.

To include the effects of stratification on turbulent mixing, Ralston et al. (2008) provided another scale of eddy viscosity that depends on the thickness of the bottom boundary layer $\hat{h}_{\mathrm{bl}}$ instead of the water depth,

$$
\hat{K}_{v}=a C_{d} \hat{U}_{T} \hat{h}_{\mathrm{bl}}
$$

where $\hat{K}_{v}$ represents the tidally averaged vertical eddy viscosity and diffusivity (assume Schmidt number of 1 ), $a$ is a constant parameter, $C_{d}$ is the bottom drag coefficient, $\hat{U}_{T}$ is the tidal current amplitude, and all variables are dimensional quantities. The bottom boundary layer $h_{\mathrm{bl}}$ can be scaled as (Stacey and Ralston 2005)

$$
\hat{h}_{\mathrm{bl}}=\hat{h}\left(\frac{R_{f}}{\mathrm{Ri}_{x}}\right)^{1 / 2} \text {, }
$$

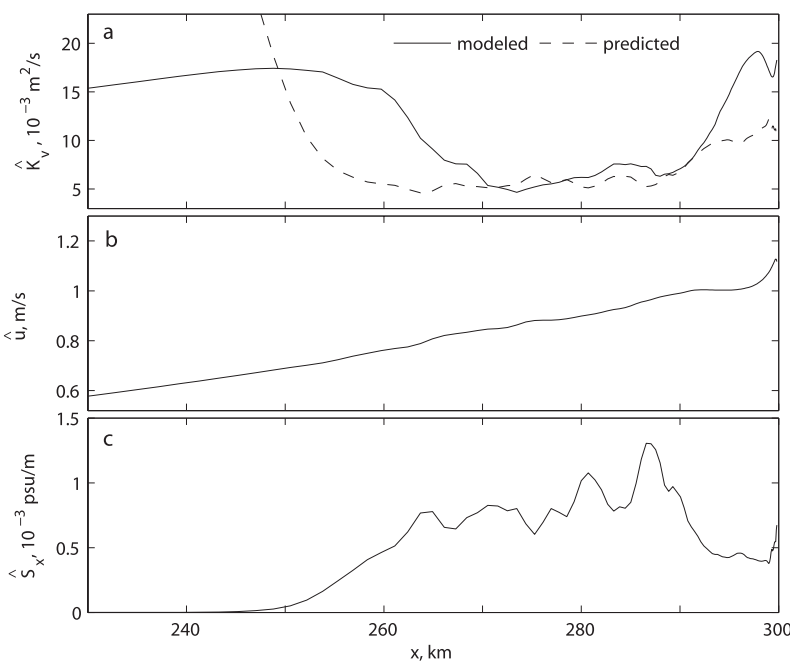

FIG. 10. Longitudinal distributions of (a) tidally averaged depthmean eddy viscosity, (b) tidal current amplitude, and (c) horizontal salinity gradient from the numerical model. Solid lines are numerical results; dashed lines are predictions using the scaling of eddy viscosity [Eq. (12)].

where $R_{f}$ is the critical flux Richardson number (taken as 0.2 here) and $\mathrm{Ri}_{x}$ is the horizontal Richardson number. The scale for the boundary layer thickness is obtained for stratified ebb tides assuming that the buoyancy flux due to straining of $d \hat{s} / d \hat{x}$ is balanced by shear production at the top of the bottom boundary layer (Stacey and Ralston 2005). The assumption involved in its derivation limits the scale to stratified water columns; $\mathrm{Ri}_{x}$ is a scale of the Richardson number by representing the vertical density difference as a function of the horizontal density gradient. Stacey et al. (2001) proposed

$$
\mathrm{Ri}_{x}=\frac{g \beta \hat{h}^{2}}{\hat{u}_{*}^{2}} \frac{\partial \hat{s}}{\partial \hat{x}}
$$

where $\hat{u}_{*}$ is the friction velocity and is related to the tidal current velocity $\hat{U}_{T}$ by $\hat{u}_{*}=\sqrt{C_{d}} \hat{U}_{T}$. To apply Eq. (12), the values of $\hat{U}_{T}, \hat{h}$, and $d \hat{s} / d \hat{x}$ were taken from the numerical experiments (Figs. 10b,c); the $C_{d}$ used was 0.0025 ; and the best fitted $a$ was 0.31 . The values of $\hat{K}_{v}$ obtained with Eq. (12) generally match the trend of the modeled eddy viscosity in the central and outer regimes of the estuary (Fig. 10a). Particularly, $\hat{K}_{v}$ exhibits approximately a constant value in the central regime. This indicates that spatially constant vertical mixing arises from the combined effect of tidal mixing and stratification. Because the influence of stratification on eddy viscosity is represented with an along-channel salinity gradient, the longitudinal distribution of the scaled eddy viscosity is approximately a mirror image of the salinity 
gradient: that is, $\hat{K}_{v} \propto(\partial \hat{S} / \partial \hat{x})^{-1 / 2}$. As the along-channel salinity gradient tends to zero in the inner regime of the estuary, the scaled eddy viscosity becomes very large and unreliable. Although Eq. (12) can be calibrated to approximate the constant turbulent mixing in the central regime of the estuary, distinct discrepancies between scaled and modeled eddy viscosity appear in the outer and inner regimes of the estuary. These discrepancies indicate that the parameterization of estuarine turbulent mixing still needs further study.

\section{Conclusions}

This study examines residual currents induced by asymmetric tidal mixing. Neglecting lateral processes and effects of the earth's rotation and assuming a weakly stratified estuary that can be approximated as a weakly nonlinear system, a two-dimensional analytical model is established. Analytical solutions show that the residual flow induced by asymmetric tidal mixing is determined by tidal currents and by the asymmetry of tidal mixing between flood and ebb. The magnitude of tidal-asymmetryinduced flow is the same order as that of density-driven flow. Typical tidal asymmetry in mixing leads to a twolayer residual flow similar in structure to the densitydriven flow, as previously established (Jay and Musiak 1996; Stacey et al. 2001). However, unlike the densitydriven flow, which has a maximum at the region of greatest horizontal density gradient, the strongest residual currents induced by tidal asymmetry appear at the estuary mouth where the maximum tidal current amplitudes are found. Reverse tidal asymmetry produces a two-layer structure that is opposite to that of the densitydriven flow.

Numerical experiments support the longitudinal pattern of the currents induced by tidal asymmetry as predicted by the analytical model. However, the modeled tidal-asymmetry-induced flow has maximum magnitudes in the central regime of the estuary where turbulent mixing is reduced. This indicates that the strength of tidal-asymmetry-induced flow is also dependent on the tidal mean eddy viscosity. The spatially constant eddy viscosity in the central regime of the estuary is found to be influenced by stratification, which is represented by the along-channel density gradient. This demonstrates that turbulent mixing is highly related to stratification in stratified estuaries.

Because this study concentrates mainly on weakly stratified estuaries, the residual currents induced by asymmetric tidal mixing in partially mixed and highly stratified estuaries are yet to be explored. The linear decomposition of residual flow used in the analytical model could be extended to partially mixed and highly stratified estuaries. Such extension will allow identification of the strength and spatial patterns of the estuarine residual current components: river-induced, densitydriven, tidal-nonlinearities-induced, and tidal-asymmetryinduced flows.

Acknowledgments. This work was supported by NSF Project OCE-0726697. AVL also acknowledges the completion of this study while a Gledden Fellow at the Centre for Water Research of the University of Western Australia. We thank David Jay and an anonymous reviewer for their insightful comments that helped to improve this work.

\section{APPENDIX}

\section{The Perturbation Equations}

This appendix gives the derivation of Eqs. (5)-(8). More detailed information can be found in Ianniello (1977, 1981) and McCarthy (1993). The dimensionless equations [Eqs. (5)] are analyzed for the situation that

$\varepsilon \ll 1, \quad \gamma=O(1), \quad R=O(1), \quad K^{*}=O(1), \quad K^{\prime}=O(1)$,

and

$$
K_{x}=O(1)
$$

These are typical values for a well-mixed estuary (vertical mixing dominates over advection), where residual currents generated by tidal advection terms, baroclinic pressure gradients, and freshwater discharge are small compared to tidal currents.

Asymptotic solutions of the system can now be constructed as perturbation series in the small parameter $\varepsilon$,

$$
(u, w, \eta, s)=\left(u_{0}, w_{0}, \eta_{0}, s_{0}\right)+\varepsilon\left(u_{1}, w_{1}, \eta_{1}, s_{1}\right)+\cdots
$$

A further implication of $\varepsilon \ll 1$ is that, in the along-channel momentum balance, the baroclinic pressure gradient term can be expanded as follows:

$$
-\varepsilon \gamma \int_{z}^{\varepsilon \eta d} \frac{\partial s}{\partial x} d z^{\prime}=-\varepsilon \gamma \int_{z}^{0} \frac{\partial s}{\partial x} d z^{\prime}+\cdots
$$

As will turn out below, it suffices to analyze the dynamics up to $O(\varepsilon)$. Likewise, the boundary conditions at the free surface can be transformed to conditions at the fixed surface $z=0$ by expanding variables in a Taylor series. For example, the kinematic condition becomes up to $O(\varepsilon)$ at $z=0$ : 


$$
w+\varepsilon \eta d \frac{\partial w}{\partial z}+\cdots=\frac{\partial \eta}{\partial t}+\varepsilon u \frac{\partial \eta}{\partial x}+\cdots
$$

To lowest order this yields the equations

$$
\begin{gathered}
\frac{\partial u_{0}}{\partial t}=-\frac{\partial \eta_{0}}{\partial x}+\frac{1}{2} \frac{\partial^{2} u_{0}}{\partial z^{2}}, \\
\frac{\partial u_{0}}{\partial x}+\frac{\partial w_{0}}{\partial z}=0 \\
\frac{\partial \eta_{0}}{\partial t}+\frac{1}{d} \frac{\partial}{\partial x}\left(\int_{-d}^{0} u_{0} d z\right)=0
\end{gathered}
$$

and

$$
\frac{\partial s_{0}}{\partial t}=\frac{1}{2} \frac{\partial^{2} s_{0}}{\partial z^{2}}
$$

The corresponding boundary conditions are

$$
\begin{aligned}
& \text { at } z=0: \quad w_{0}=\frac{\partial \eta_{0}}{\partial t}, \quad \frac{\partial u_{0}}{\partial z}=0, \quad \frac{\partial s_{0}}{\partial z}=0 \\
& \text { at } z=-d: \quad w_{0}=0, \quad u_{0}=0, \quad \frac{\partial s_{0}}{\partial z}=0 \\
& \text { at } x=0: \quad \eta_{0}=\cos (\omega t), \quad s_{0}=1
\end{aligned}
$$

and

$$
\text { at } x \rightarrow \infty: \quad \frac{1}{d} \int_{-d}^{0} u_{0} d z \rightarrow 0, \quad \frac{1}{d} \int_{-d}^{0} u_{0} s_{0} d z \rightarrow 0 .
$$

This system describes a damped linear tidal wave that travels into the estuary. The equation of $s_{0}$ is a diffusion equation. Considering the boundary conditions of $s_{0}$, for any initial conditions variations in $s_{0}$ over the vertical will become smaller in the course of time; thus, the nontransient solution of this equation is

$$
s_{0}=s_{0}(x) .
$$

Thus, to lowest order the salinity is only a function of the along-channel coordinate $x$, as is to be expected for a well-mixed or weakly stratified estuary (in reality, $s_{0}$ may vary with time and depth in weakly stratified estuaries, so the assumption needs further study). To determine $s_{0}$, tidally averaging and depth integrating the salt transport equation $(5 \mathrm{~d})$ gives

$$
\overline{\int_{-d}^{\varepsilon \eta d}\left(u s-\varepsilon K_{x} \frac{\partial s}{\partial x}\right) d z}=0 .
$$

The lowest order equation of the asymptotic expansion of Eq. (A8) is

$$
\frac{1}{d} \int_{-d}^{0} \overline{u_{0} s_{1}} d z-R s_{0}-K_{x} \frac{d s_{0}}{d x}=0
$$

where $R$ is the dimensionless river flow velocity, $O(1)$. This equation reveals a balance between tide-induced salt transport, a term owing to freshwater transport and longitudinal dispersion. The first-order equations can be derived in a straightforward manner. The final equations contain quite a number of terms, but some of them can be removed because it is known that $s_{0}$ only depends on $x$. The resulting equations in this case read as

$$
\begin{aligned}
\frac{\partial u_{1}}{\partial t}+u_{0} \frac{\partial u_{0}}{\partial x}+w_{0} \frac{\partial u_{0}}{\partial z}= & -\frac{\partial \eta_{1}}{\partial x}+\gamma \frac{d s_{0}}{d x} z+\frac{1}{2} \frac{\partial^{2} u_{1}}{\partial z^{2}} \\
& +\frac{1}{2} \frac{\partial}{\partial z}\left(K^{\prime} \frac{\partial u_{0}}{\partial z}\right),
\end{aligned}
$$

$$
\frac{\partial u_{1}}{\partial x}+\frac{\partial w_{1}}{\partial z}=0,
$$

$$
\frac{\partial \eta_{1}}{\partial t}+\frac{1}{d} \frac{\partial}{\partial x}\left(\int_{-d}^{0} u_{1} d z\right)+\frac{\partial}{\partial x}\left(\left.u_{0}\right|_{z=0} \eta_{0}\right)=0
$$

and

$$
\frac{\partial s_{1}}{\partial t}+u_{0} \frac{\partial s_{0}}{\partial x}=\frac{1}{2} \frac{\partial^{2} s_{1}}{\partial z^{2}}
$$

with corresponding boundary conditions

$$
\begin{gathered}
\text { at } z=0: \quad w_{1}=\frac{\partial \eta_{1}}{\partial t}+u_{0} \frac{\partial \eta_{0}}{\partial x}, \\
\frac{\partial u_{1}}{\partial z}+d \eta_{0} \frac{\partial^{2} u_{0}}{\partial z^{2}}+K^{\prime} \frac{\partial u_{0}}{\partial z}=0, \\
\frac{\partial s_{1}}{\partial z}=0 ; \\
\text { at } z=-d: \quad w_{1}=0, \quad u_{1}=0, \quad \frac{\partial s_{1}}{\partial z}=0 ; \\
\text { at } x=0: \quad \eta_{1}=0, \quad s_{1}=0 ;
\end{gathered}
$$

and

$$
\text { at } x \rightarrow \infty: \quad \frac{1}{d} \int_{-d}^{0} u_{1} d z \rightarrow-R, \quad \frac{1}{d} \int_{-d}^{0} u_{0} s_{1} d z \rightarrow 0 .
$$

It is noticed that asymmetric tidal mixing in this model only enters in the momentum balance, and not in the salt balance. This is due to our assumption of strong vertical 
mixing, such that $s_{0}$ does not depend on the vertical coordinate. The focus of this paper is on the residual components for which the equations are obtained by averaging all first-order equations over a tidal cycle. These can subsequently be solved. In this study, the new aspect concerns the solution of the residual flow induced by time-varying tidal mixing as caused by the eddy viscosity coefficient $K^{\prime}$. Owing to adopting a constant $\hat{K}^{*}$, the factor of shear stress has been changed from $3 / 8$ in Ianniello (1977) and McCarthy (1993) to $1 / 2$ in this study. The solution to $\bar{u}_{1 N}$ is the same as that of Ianniello (1977, 1981), which neglected the factor $3 / 4$ for the case with constant eddy viscosity, whereas the solution to $\bar{u}_{1 D}$ from McCarthy (1993) has been changed to

$$
\bar{u}_{1 D}=\frac{\gamma d^{3}}{24} \frac{d s_{0}}{d x}\left(1-9 \frac{z^{2}}{d^{2}}-8 \frac{z^{3}}{d^{3}}\right)
$$

The solution to $\bar{u}_{1 R}$ was not affected by the factor $3 / 4$.

\section{REFERENCES}

Businger, J. A., J. C. Wyngaard, Y. Izumi, and E. F. Bradley, 1971: Flux profile relationships in the atmospheric surface layer. J. Atmos. Sci., 28, 181-189.

Chatwin, P. C., 1976: Some remarks on the maintenance of the salinity distribution in estuaries. Estuarine Coastal Mar. Sci., 4, $555-566$.

Cheng, P., and A. Valle-Levinson, 2009: Influence of lateral advection on residual currents in microtidal estuaries. J. Phys. Oceanogr., 39, 3177-3190.

— , R. E. Wilson, R. J. Chant, D. C. Fugate, and R. D. Flood, 2009: Modeling influence of stratification on lateral circulation in a stratified estuary. J. Phys. Oceanogr., 39, 2324-2337.

Fram, J. P., M. Martin, and M. T. Stacey, 2007: Exchange between the coastal ocean and a semi-enclosed estuarine basin: Dispersive fluxes. J. Phys. Oceanogr., 37, 1645-1660.

Fugate, D. C., C. T. Friedrichs, and L. P. Sanford, 2007: Lateral dynamics and associated transport of sediment in the upper reaches of a partially mixed estuary, Chesapeake Bay, USA. Cont. Shelf Res., 27, 679-698.

Haidvogel, D. B., H. G. Arango, K. Hedstrom, A. Beckmann, P. Malanotte-Rizzoli, and A. F. Shchepetkin, 2000: Model evaluation experiments in the North Atlantic basin: Simulations in nonlinear terrain-following coordinates. Dyn. Atmos. Oceans, 32, 239-281.

Hansen, D. V., and M. Rattray, 1965: Gravitational circulation in straits and estuaries. J. Mar. Res., 23, 104-122.

Hetland, R. D., and W. R. Geyer, 2004: An idealized study of the structure of long, partially mixed estuaries. J. Phys. Oceanogr., 34, 2677-2691.

Huijts, K. M. H., H. M. Schuttelaars, H. E. de Swart, and C. T. Friedrichs, 2009: Analytical study of the transverse distribution of along-channel and transverse residual flows in tidal estuaries. Cont. Shelf Res., 29, 89-100.
Ianniello, J. P., 1977: Tidally induced residual currents in estuaries of constant breadth and depth. J. Mar. Res., 35, 755-786.

_ 1981: Comments on tidally induced residual currents in estuaries: Dynamics and near bottom flow characteristics. J. Phys. Oceanogr., 11, 126-134.

Jay, D. A., and J. D. Smith, 1990: Residual circulation in shallow estuaries. 2. Weakly stratified and partially mixed, narrow estuaries. J. Geophys. Res., 95, 733-748.

__ and J. M. Musiak, 1996: Internal tidal asymmetry in channel flows: Origins and consequences. Mixing in Estuaries and Coastal Seas, Geophys. Monogr., Vol. 50, Amer. Geophys. Union, 211-249.

Lacy, J. R., M. T. Stacey, J. R. Burau, and S. G. Monismith, 2003: The interaction of lateral baroclinic forcing and turbulence in an estuary. J. Geophys. Res., 108, 3089, doi:10.1029/2002JC001392.

Li, M., J. Trowbridge, and R. Geyer, 2008: Asymmetric tidal mixing due to the horizontal density gradient. J. Phys. Oceanogr., 38, 418-434.

MacCready, P., 2004: Toward a unified theory of tidally-averaged estuarine salinity structure. Estuaries, 27, 561-570.

—, 2007: Estuarine adjustment. J. Phys. Oceanogr., 37, 2133-2145.

McCarthy, R. K., 1993: Residual currents in tidally dominated, well-mixed estuaries. Tellus, 45A, 325-340.

Nepf, H. M., and W. R. Geyer, 1996: Intratidal variations in stratification and mixing in the Hudson estuary. J. Geophys. Res., 101 (C5), 12 079-12 086.

Officer, C. B., 1976: Physical Oceanography of Estuaries (and Associated Coastal Waters). John Wiley, $465 \mathrm{pp}$.

Pritchard, D. W., 1952: Salinity distribution and circulation in the Chesapeake Bay estuarine system. J. Mar. Res., 11, 106-123.

_ 1956: The dynamic structure of a coastal plain estuary. J. Mar. Res., 15, 33-42.

Ralston, D. K., W. R. Geyer, and J. A. Lerczak, 2008: Subtidal salinity and velocity in the Hudson River estuary: Observations and modeling. J. Phys. Oceanogr., 38, 753-770.

Scully, M. E., and C. T. Friedrichs, 2007: The importance of tidal and lateral symmetries in stratification to residual circulation in partially mixed estuaries. J. Phys. Oceanogr., 37, 1496-1511.

— W. R. Geyer, and J. A. Lerczak, 2009: The influence of lateral advection on the residual estuarine circulation: A numerical modeling study of the Hudson River estuary. J. Phys. Oceanogr., 39, 107-124.

Simpson, J. H., J. Brown, J. Matthews, and G. Allen, 1990: Tidal straining, density currents, and stirring in the control of estuarine stratification. Estuaries, 13, 125-132.

- H. Burchard, N. R. Fisher, and T. P. Rippeth, 2002: The semidiurnal cycle of dissipation in a ROFI: Model-measurement comparisons. Cont. Shelf Res., 22, 1615-1628.

—, E. Williamsa, L. H. Brasseurb, and J. M. Brubakerb, 2005: The impact of tidal straining on the cycle of turbulence in a partially stratified estuary. Cont. Shelf Res., 25, 51-64.

Stacey, M. T., and D. K. Ralston, 2005: The scaling and structure of the estuarine bottom boundary layer. J. Phys. Oceanogr., 35, 55-71.

, J. R. Burau, and S. G. Monismith, 2001: Creation of residual flows in a partially stratified estuary. J. Geophys. Res., 106, 17 013-17 037.

, J. P. Fram, and F. K. Chow, 2008: Role of tidally periodic density stratification in the creation of estuarine subtidal circulation. J. Geophys. Res., 113, C08016, doi:10.1029/2007JC004581. 